\title{
Di (2-ethylhexyl) phthalate exposure impairs meiotic progression and DNA damage repair in fetal mouse oocytes in vitro
}

\author{
Jing-Cai Liu ${ }^{1}$, Fang-Nong Lai ${ }^{1}$, Ling Li ${ }^{2}$, Xiao-Feng Sun ${ }^{1,3}$, Shun-Feng Cheng ${ }^{1}$, Wei Ge ${ }^{1}$, Yu-Feng Wang ${ }^{1}$, Lan Li ${ }^{1}$, Xi-Feng Zhang ${ }^{4}$, \\ Massimo De Felici ${ }^{5}$, Paul W Dyce ${ }^{6}$ and Wei Shen ${ }^{*, 1}$
}

Di (2-ethylhexyl) phthalate (DEHP), is the most common member of the class of phthalates that are used as plasticizers and have become common environmental contaminants. A number of studies have shown that DEHP exposure impacts reproductive health in both male and female mammals by acting as an estrogen analog. Here, we investigated the effects of DEHP on meiotic progression of fetal mouse oocytes by using an in vitro model of ovarian tissue culture. The results showed that 10 or $100 \mu \mathrm{M}$ DEHP exposure inhibited the progression of oocytes throughout meiotic prophase I, specifically from the pachytene to diplotene stages. DEHP possibly impairs the ability to repair DNA double-strand breaks induced by meiotic recombination and as a consequence activates a pachytene check point. At later stages, such defects led to an increased number of oocytes showing apoptotic markers (TUNEL staining, expression of pro-apoptotic genes), resulting in reduced oocyte survival, gap junctions, and follicle assembly in the ovarian tissues. Microarray analysis of ovarian tissues exposed to DEHP showed altered expression of several genes including some involved in apoptosis and gonad development. The expression changes of some genes clustered in cell-cell communication and signal transduction, along with plasma membrane, extracellular matrix and ion channel function classes, were dependent on the DEHP concentration. Together, these results bring new support to the notion that exposure to DEHP during gestation might exert deleterious effects on ovary development, perturbing germ cell meiosis and the expression of genes involved in a wide range of biological processes including ovary development.

Cell Death and Disease (2017) 8, e2966; doi:10.1038/cddis.2017.350; published online 3 August 2017

Di (2-ethylhexyl) phthalate (DEHP), is a compound largely used in plasticizing polyvinyl chloride resin (PVC) products worldwide. ${ }^{1}$ More than 10 million tons of DEHP is estimated to be used in the production of plastics and plastic-based products every year. ${ }^{2}$ DEHP, due to its weak non covalent link to plastic components, can be easily released into the environment and humans and animals are exposed through oral ingestion, inhalation and skin contact, after which it enters into the blood circulation. ${ }^{3}$

Previous studies have indicated that DEHP exposure is associated with testicular, liver, kidney and ovary tissue disease. $^{3-7}$ The modes of action of DEHP are not well understood. Mechanisms may include activation of peroxisome proliferator-activated receptors (PPARs), estrogen receptors (ERs) and to a lesser extent androgen receptors (ARs). PPARs are a group of nuclear receptor proteins that function as transcription factors for genes involved in a variety of cell activities including steroidogenesis and antioxidative actions. ERs are receptors that are activated by estrogens, in particular $17 \beta$-estradiol (E2). Two classes of ERs have been described: nuclear/cytoplasmic estrogen receptors ERa and $E R \beta$, and membrane estrogen receptors (mERs). Although the expression and role of PPARs in the mammalian ovary before birth are not known, ERa is known to be expressed by both primordial germ cells (PGCs) ${ }^{8}$ and somatic cells ${ }^{9}$ within sexually undifferentiated mouse gonads. DEHP effects on early postnatal and adult ovaries appear to be a consequence of activation of PPAR and/or ER-dependent pathways. It is apparent that DEHP effects on oogenesis are strictly dependent on the period of exposure, both in vivo and in vitro. Effects are seen during several developmental waypoints including during the formation and development of PGCs, entrance and progression of oocytes into meiotic prophase I, germ cell cyst breakdown during the prenatal or early postnatal period, along with primordial follicle activation and development in the adult ovary. ${ }^{10,11}$

In the present paper, we utilized an in vitro ovarian tissue culture system, developed in our lab, to study the effects of DEHP on prenatal or early postnatal oogenesis. ${ }^{12}$ While it has been reported that DEHP effects folliculogenesis through impairment of oocyte meiosis, survival, and follicle assembly, ${ }^{10}$ the mechanisms involved remain largely unknown. Using our in vitro system we investigated the mechanisms of such effects and identified the gene expression profile induced by DEHP in the fetal ovary.

\footnotetext{
${ }^{1}$ College of Animal Science and Technology, Institute of Reproductive Sciences, Qingdao Agricultural University, Qingdao 266109, China; ${ }^{2}$ Tengzhou People's Hospital, Tengzhou 277500, China; ${ }^{3}$ College of Life Sciences, Qingdao Agricultural University, Qingdao 266109, China; ${ }^{4}$ College of Biological and Pharmaceutical Engineering, Wuhan Polytechnic University, Wuhan 430023, China; ${ }^{5}$ Department of Biomedicine and Prevention, University of Rome Tor Vergata, Rome 00133 , Italy and ${ }^{6}$ Department of Animal Sciences, College of Agriculture, Auburn University, Auburn, AL 36849, USA

${ }^{*}$ Corresponding author: W Shen, College of Animal Science and Technology, Institute of Reproductive Sciences, Qingdao Agricultural University, Qingdao 266109 , China. Tel: +860 532880302 46; Fax: +860 532860807 73; E-mail: wshen@qau.edu.cn or shenwei427@163.com

Received 19.1.17; revised 26.6.17; accepted 02.7.17; Edited by M Agostini
} 

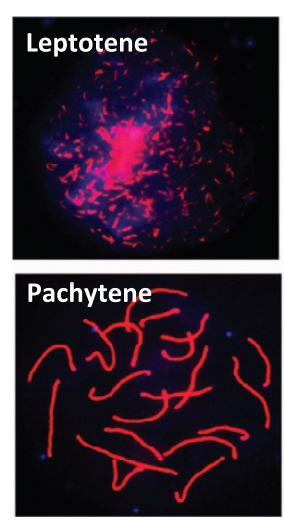

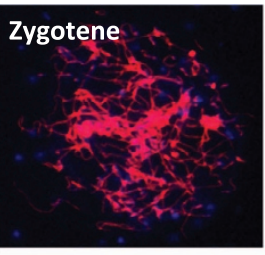

Diplotene

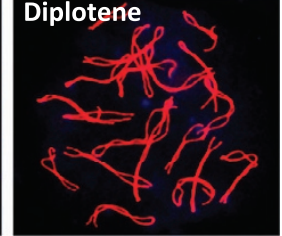

b

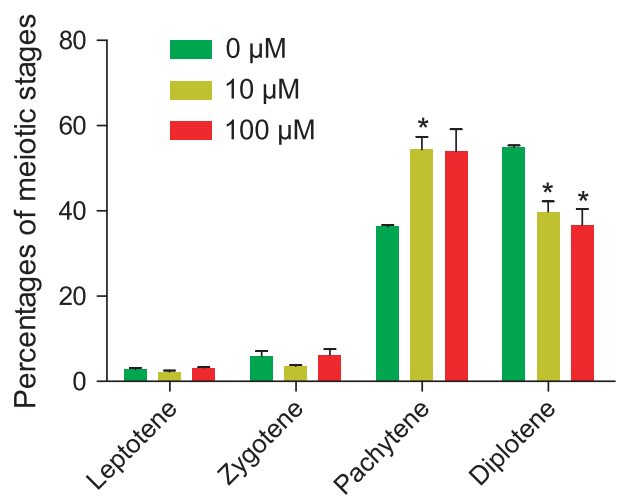

。

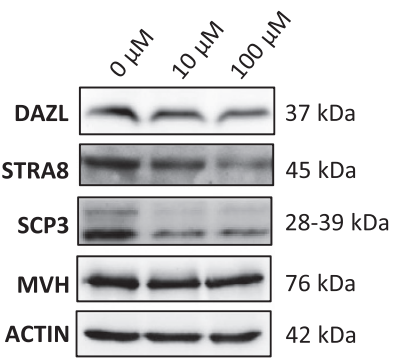

DAZL

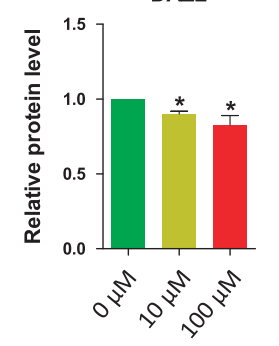

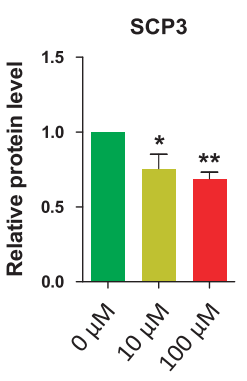

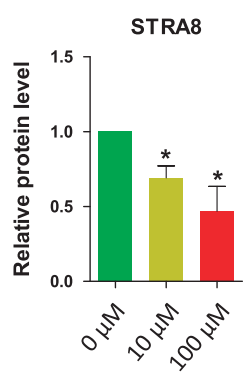

d
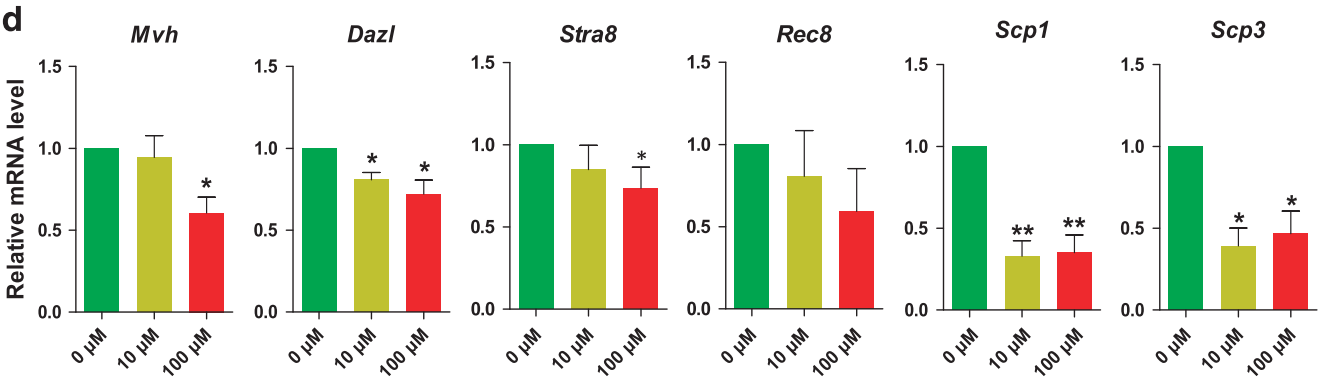

Figure 1 Exposure to DEHP impairs meiotic progression of oocytes from pachytene to diplotene. (a) Immunolabeling of the oocyte chromosomes with anti-SYCP3 antibody (red) and Hoechst 33342 (blue). (b) Effect of DEHP on meiotic progression of oocytes throughout prophase I stages; percentage of each group is presented as mean \pm SD. Control: $36.27 \pm 0.80 \%$ pachytene and $54.99 \pm 0.66 \%$ diplotene; $10 \mu \mathrm{M}$ and $100 \mu \mathrm{M}$ DEHP $57.79 \pm 4.22 \%$ and $56.62 \pm 6.62 \%$ pachytene and $39.79 \pm 4.22 \%$ and $36.62 \pm 6.62 \%$ diplotene, respectively. (c) Representative WB showing the effect of DEHP on the expression of germ cell (DAZL) and meiotic (STRA8 and SCP3) Specific proteins. (d) Effect of DEHP on the levels of mRNA in the ovarian tissues of germ cell (Mvh and Dazl), meiotic (Stra8, Rec8, Scp1 and Scp3). All experiments were repeated at least three times independently. ( $\left.{ }^{*} P<0.05 ;{ }^{* \star} P<0.01\right)$

\section{Results}

Exposure of the ovary to DEHP delays oocyte progression from pachytene to diplotene. In order to explore the effects of DEHP exposure on the progression of meiotic prophase I in female germ cells, $12.5 \mathrm{dpc}$ fetal mouse ovarian tissues were cultured in vitro and exposed to DEHP for 6 days as the first meiotic prophase I accomplished at $18.5 \mathrm{dpc}$ in vivo, relative to day 6 in vitro (Supplementary Figure S1). Cytospread immunofluorescence staining of meiotic chromosomes for SCP3 revealed a clear delay in meiotic progression from the pachytene to the diplotene stage in oocytes cultured in the presence of $10 \mu \mathrm{M}$ or $100 \mu \mathrm{M} \mathrm{DEHP} \mathrm{(Figures} 1 \mathrm{a}$ and $\mathrm{b}$ ). Western blot and RT-qPCR analysis showed that after 2 days of culture the expression of germ cell and meiotic genes such as Dazl and Mvh or Stra8, Scp1, Scp3 and Rec8, respectively, were significantly decreased at both at the protein and/or mRNA level (Figures 1c and d).

DEHP exposure affects the patterns of $\gamma \mathrm{H} 2 \mathrm{AX}$ and causes DNA damage in oocytes. Since the DEHPinduced meiotic delay reported above resembles the meiotic arrest occurring in a variety of species as a consequence of the pachytene checkpoint triggered by defects in DNA double-strand break (DSB) repair, ${ }^{13}$ we decided to investigate the status of DNA breaks and repair in DEHP treated oocytes.

As shown in Figure 2a, we observed that in both the control and DEHP treated oocytes the staining pattern of $\gamma \mathrm{H} 2 \mathrm{AX}$ (a marker of DNA breaks) could be classified into three main categories: negative (none or rare barely detectable foci), weak (a few number of small foci) and strong (numerous small 
a
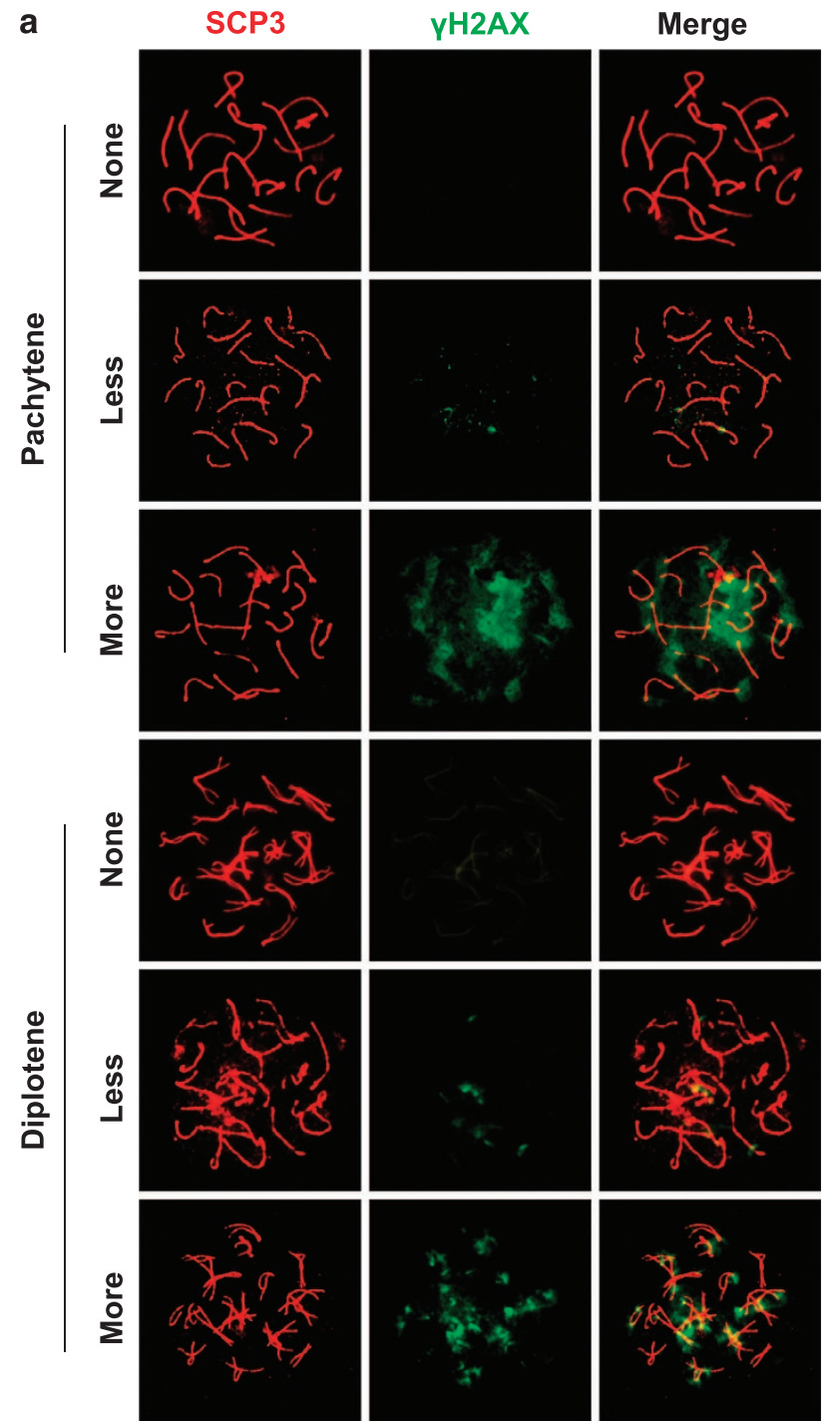
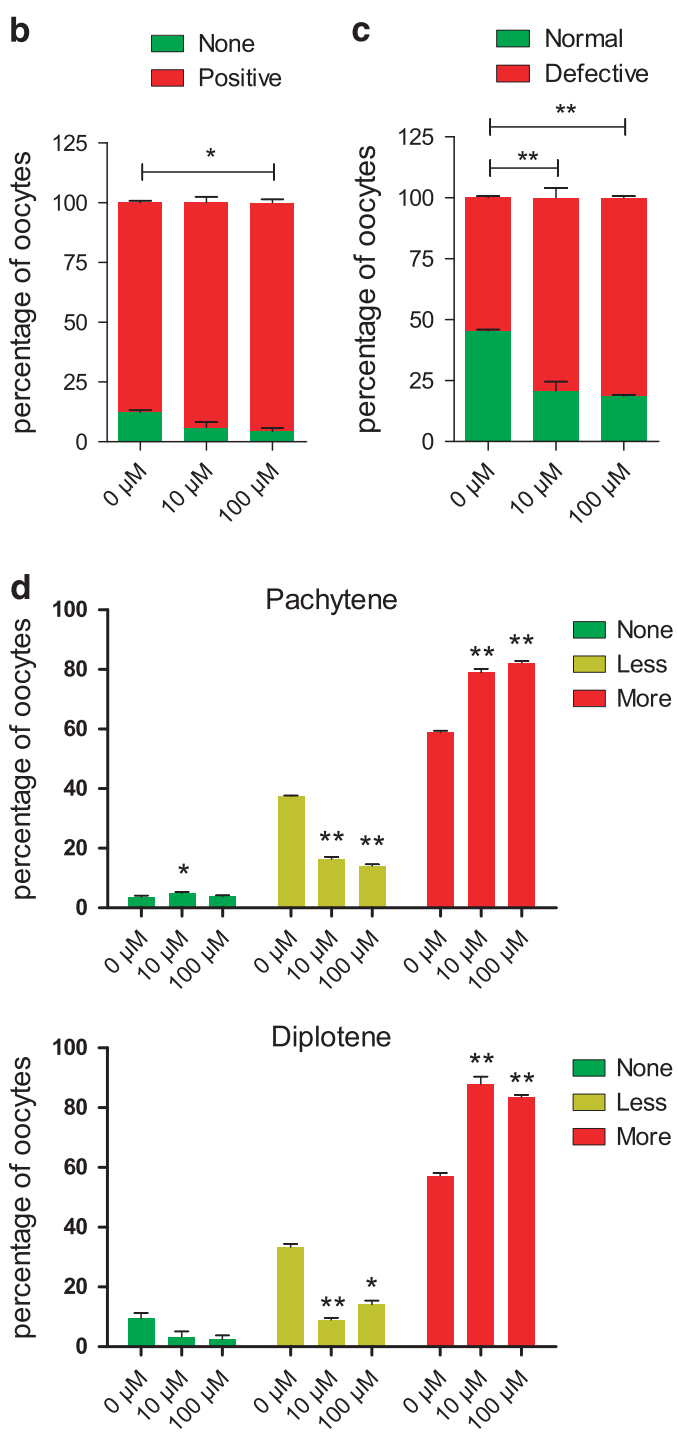

Figure 2 Effect of DEHP on $\gamma \mathrm{H} 2 \mathrm{AX}$ pattern. (a) Immunolabeling of the oocyte chromosomes with anti-SYCP3 (red) and anti- $\gamma \mathrm{H} 2 \mathrm{AX}$ (green) antibodies. (b) Percentage of positive $\gamma \mathrm{H} 2 \mathrm{AX}$ oocytes $(79.36 \pm 3.97 \%$ and $81.40 \pm 0.62 \%$, respectively), were significantly higher than that of control group $(54.70 \pm 0.61 \% ; P<0.01)$. (c) Percentage of normal, negative or weak $\gamma \mathrm{H} 2 \mathrm{AX}$ staining and defective (strong $\gamma \mathrm{H} 2 \mathrm{AX}$ staining) oocytes after six days of culture with or without DEHP. (d) Percentage of oocytes at the pachytene and diplotene stages showing negative, weakly and strongly positive $\gamma \mathrm{H} 2 \mathrm{AX}$ staining after six days of culture with or without $\mathrm{DEHP}$. ( ${ }^{\star} P<0.05 ;{ }^{* \star} P<0.01$ )

and large foci). As shown in Figure $2 \mathrm{~b}$ following 6 days of culture, the percent of $\gamma \mathrm{H} 2 \mathrm{AX}$-positive oocytes (including weak and strong staining) was higher in DEHP treated oocytes in comparison to the control, although significantly different only in the $100 \mu \mathrm{M}$ DEHP treatment group. However, comparing negative and weak oocytes against strong $\gamma \mathrm{H} 2 \mathrm{AX}$ stained oocytes, the difference was significant at both DEHP concentrations (Figure 2c). This difference was evident also when the analysis was restricted to pachytene and diplotene oocytes, in which the frequency of strong $\gamma \mathrm{H} 2 \mathrm{AX}$ staining was much higher both in control and treated oocytes (Figure 2d). The presence of large $\gamma \mathrm{H} 2 \mathrm{AX}$ foci in the DEHP exposed oocytes potentially reflects abnormal chromatin modifications during late prophase I or alternatively, a failure to resolve DSBs, and therefore a defect in DNA repair.

Evidence of increased number of oocytes with DNA damage following DEHP exposure, were also obtained by performing chromosome staining for the proteins BRCA1, usually recruited with $\mathrm{\gamma H} 2 \mathrm{AX}$ to sites of DNA breaks (Figure 3$),{ }^{14}$ and RAD51, involved in DNA repair by homologous recombination (Figure 4). Since RAD51 foci, indicative of DNA damage repair, normally disappears at the pachytene/diplotene stage oocytes at these stages still showed positive RAD51 staining both as discrete or lined dots, can be considered defective. On the other hand, the chromosome staining for the cross over protein MLH1 was not significantly different between the control and the experimental groups (Supplementary Figure S2).

Western blot results of $\gamma \mathrm{H} 2 \mathrm{AX}$ depicted in Supplementary Figure S3A show that exposure to $10 \mu \mathrm{M}$ and $100 \mu \mathrm{M}$ DEHP for 6 days caused increased expression of $\gamma \mathrm{H} 2 \mathrm{AX}$ in the ovarian tissue (Figure 2). Moreover, RT-qPCR analysis showed that exposure to $10 \mu \mathrm{M}$ and $100 \mu \mathrm{M}$ DEHP for 6 days caused increased expression of Rad51, and Brca1 transcripts 

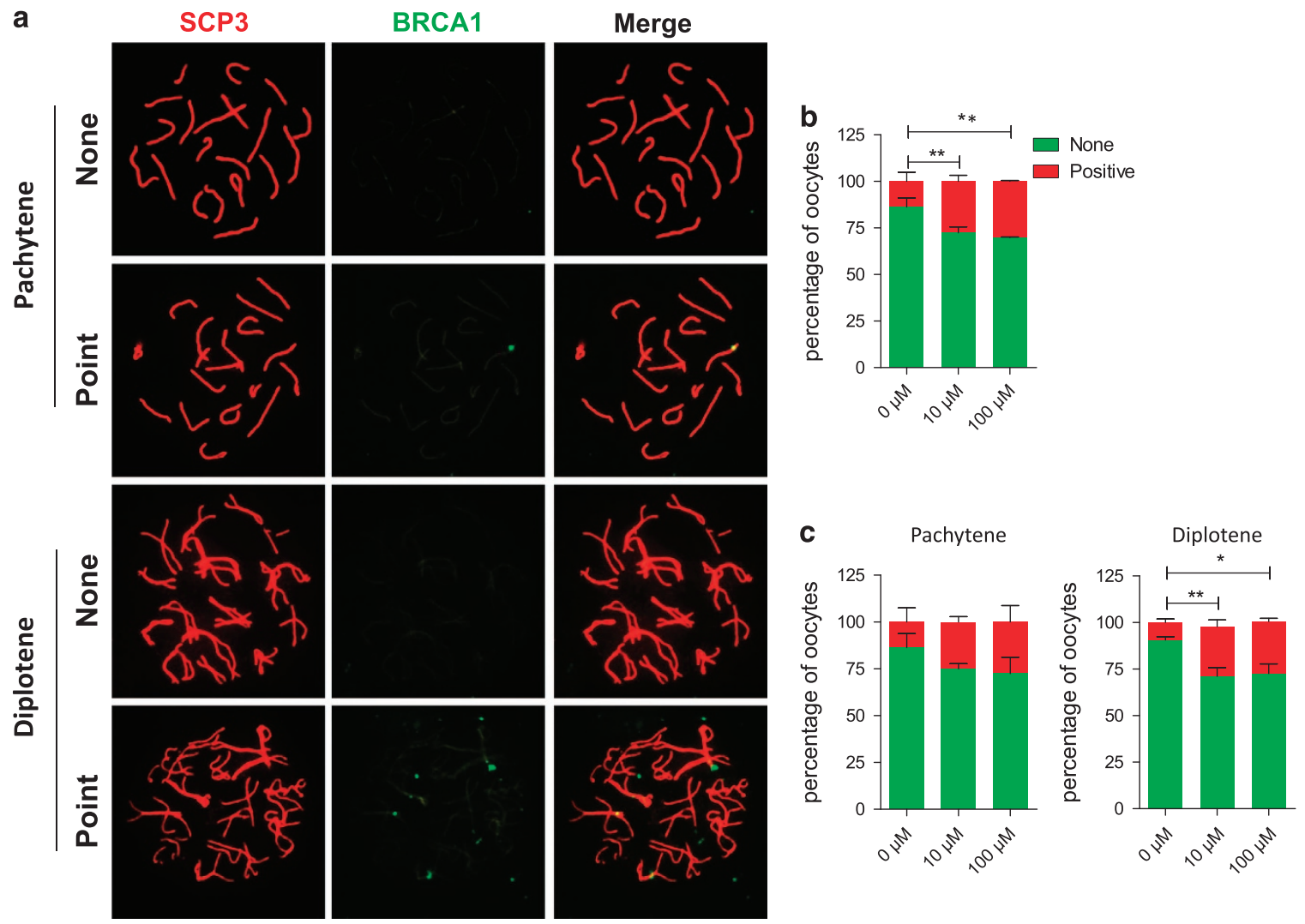

Figure 3 Exposure to DEHP impairs DNA repair in oocytes. (a) Immunolabeling of the oocyte chromosomes with anti-SYCP3 (red) and anti-BRCA1 (green) antibodies. (b) Percentage of BRCA1 positive oocytes after six days of culture with or without DEHP (c) Percentage of oocytes at the pachytene and diplotene stages showing negative or positive BRCA1 staining after 6 days of culture with or without DEHP. ( $\left.{ }^{*} P<0.05 ;{ }^{* \star} P<0.01\right)$

along with Mlh1 and Spo11 genes, the latter encoding a protein involved in the creation of DSB in the DNA at the preleptotene/leptotene stage (Supplementary Figure S3C). ${ }^{15}$ Finally the level of mRNA of the Atm gene encoding a protein that is recruited and activated by DNA breaks was not significantly altered while the expression of the p53 gene, a gene essential for chromosome stability, ${ }^{16}$ was decreased both at the mRNA and protein level (Supplementary Figure S3B-C), further indicating that DEHP exposed cells accumulated DNA damage.

DEHP promotes the expression of ERs and decreases the expression of PPARa. In order to examine the expression of ERs and PPARa, two possible DEHP targets, we analyzed ovarian tissues by RT-qPCR. The analysis showed that the exposure to $10 \mu \mathrm{M}$ and $100 \mu \mathrm{M}$ DEHP for 6 days caused significantly increased mRNA expression of $E R a$ and $E R \beta$, and decreased the expression of PPARa. Such effects were partly abolished by the contemporaneous addition to the culture of $1 \mu \mathrm{M}$ tamoxifen, an ER antagonist (Figure 5a). Western blot results confirmed the increased expression of the ERa protein after exposure to $10 \mu \mathrm{M}$ and $100 \mu \mathrm{M}$ DEHP, and the inhibitory effect by $1 \mu \mathrm{M}$ tamoxifen (Figure $5 \mathrm{~b}$ ). Furthermore, the percentage of cells showing PPARa positive staining was significantly decreased by treatment with DEHP in comparison to controls (Figure 5 c).
DEHP exposure induces apoptosis in ovarian cells. Since a major consequence of unrepaired DNA damage is cell apoptosis, we analyzed ovarian tissues incubated in the presence of DEHP for 6 days for apoptotic markers. The number of cells showing TUNEL-positive staining was significantly increased by DEHP treatment when compared to the control (Figure 6a; Supplementary Figure S4). Moreover, as shown in Figure $6 \mathrm{~b}$, the ratio of the $\mathrm{Bax} / \mathrm{BCl}-2$ transcripts and the level of mRNA for the Caspase3 gene were significantly increased in the DEHP exposed ovaries. Western blot analyses of the ovarian tissue confirmed the increased ratio of $\mathrm{Bax} / \mathrm{BCl}-2$ at protein level (Figure 6c), and, in addition, showed an apparent paradoxically increased expression of the anti-apoptotic protein MCL-1 after DEHP treatment in comparison to control (Figures 6d and e; Supplementary Figure S3D).

TEM images displayed some apoptotic characteristics in oocytes following DEHP treatment. While control oocytes showed a regular nuclear membrane with homogeneously dispersed chromatin and normal cytoplasmic structures, oocytes in DEHP treated groups showed condensed chromatin regions (Supplementary Figure S5A). Furthermore, numerous myelin bodies and dark homogenous osmophilic lipid droplets with distinct lamellae were observed in the $100 \mu \mathrm{M}$ DEHP treated oocytes (Supplementary Figure S5B). 
a

(
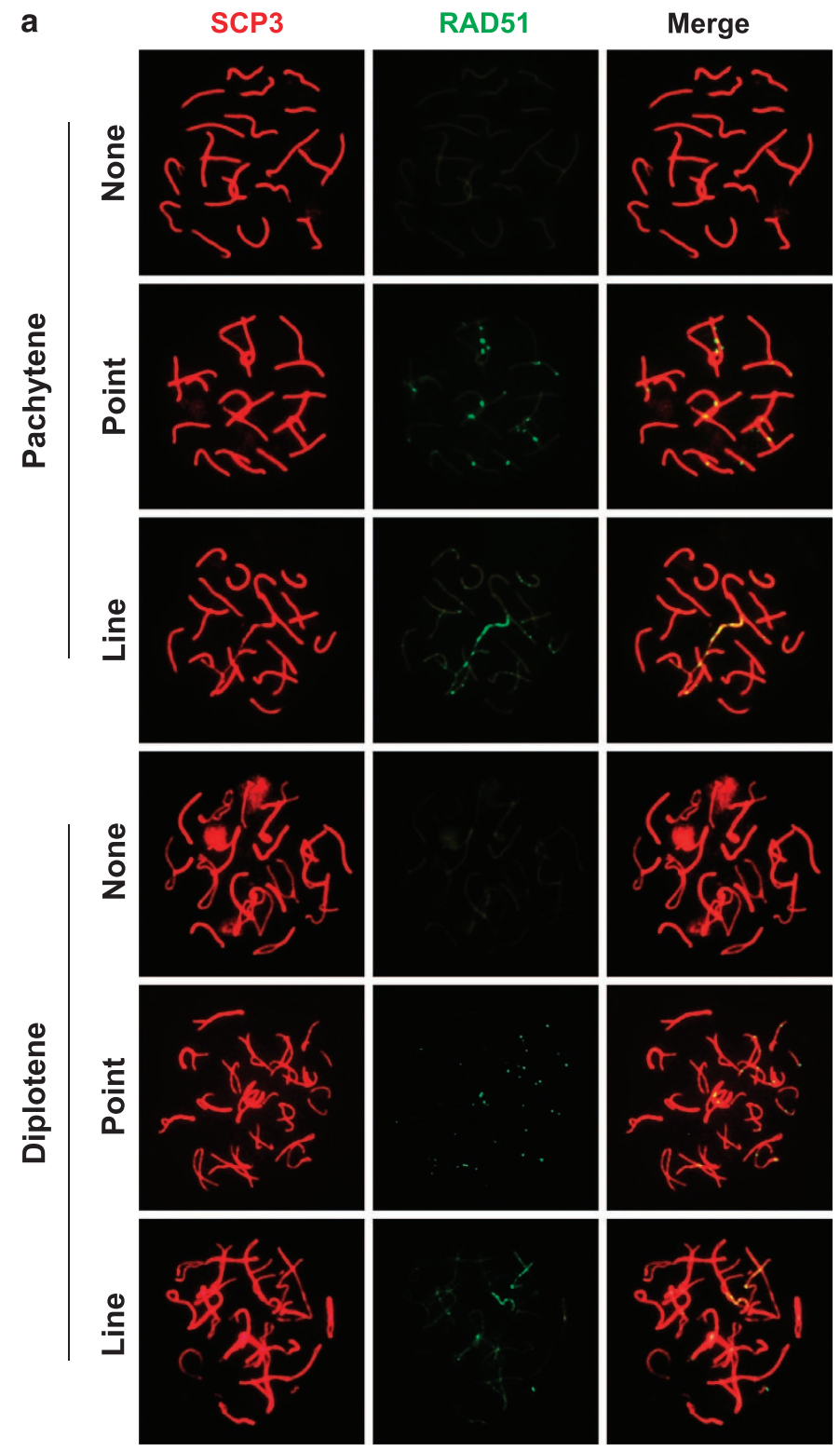
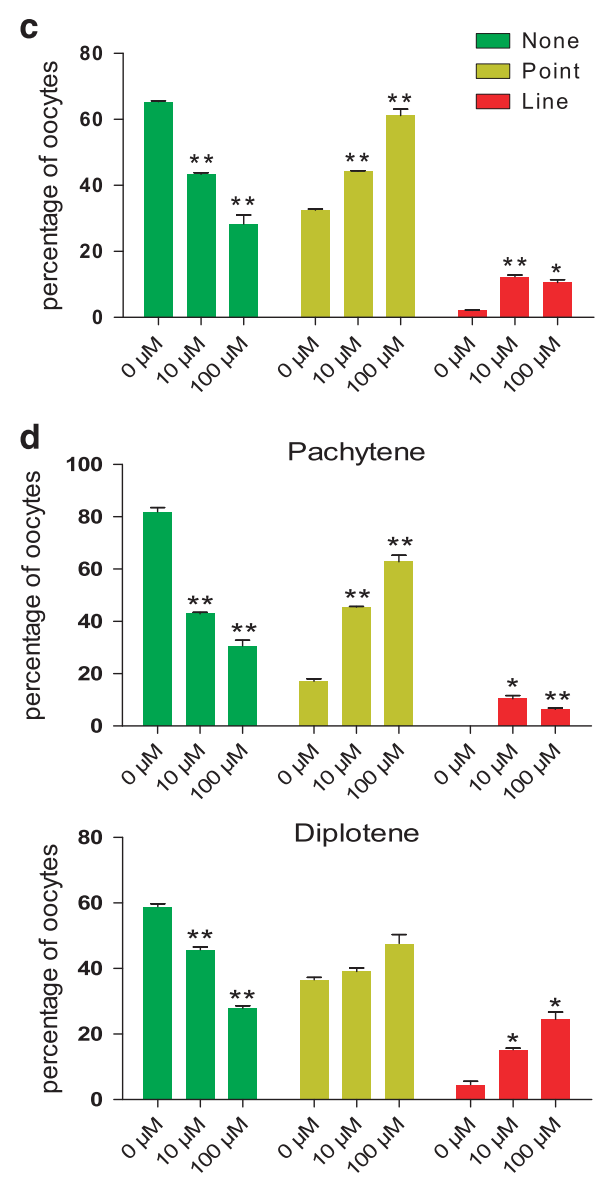

Figure 4 Exposure to DEHP impairs DNA repair in oocytes. (a) Immunolabeling of the oocyte chromosomes with anti-SYCP3 (red) and anti-RAD51 (green) antibodies. (b) Percentage of oocytes showing none, RAD51 staining after six days of culture with or without DEHP. (c) Percentage of oocytes showing none, point or line RAD51 staining after six days of culture with or without DEHP. (d) Percentage of oocytes at the pachytene and diplotene stages showing none, point or line RAD51 staining after six days of culture with or without DEHP $\left({ }^{*} P<0.05 ;{ }^{* *} P<0.01\right)$

DEHP exposure reduces the number of oocytes, decreases the expression of gap junction proteins and inhibits cyst breakdown. By prolonging the culture up to 10 days we observed additional effects of DEHP exposure on the ovarian tissues. DEHP exposure caused a significant reduction in the number of oocytes (Control $=258.67 \pm$ $31.5 \% ; 10 \mu \mathrm{M}$ DEHP $=202.33 \pm 49.5 \%$ and $100 \mu \mathrm{M}$ DEHP $=$ $124.83 \pm 28.86 \%$, Figures $7 a$ and $b$ ).

Furthermore, a survey of the distribution of gap junctions using an antibody against $\mathrm{Cx} 43$, known to form channels mostly among granulosa cells, showed a decreased number of gap junctional plaques in the ovarian fragments incubated in the presence of DEHP when compared to the control unexposed fragments (Control $=94.50 \pm 20 ; 10 \mu \mathrm{M}$ DEHP $=$ $54.25 \pm 8.26$; $100 \mu \mathrm{M}$ DEHP $=34.75 \pm 8.14$; Figures $7 \mathrm{~d}$ and e). RT-qPCR analyses showed a DEHP dependent reduction not only of $C x 43$ expression but also of the $C \times 37$ gene, encoding a connexin forming gap junction channels between granulosa cells and the oocyte (Figure 7f).

Finally, numerous germ cell nests and rare follicles were detected within the ovarian tissues in the presence of DEHP (Figure 7c). Follicle counts confirmed a marked reduction in the follicle number and higher percentage of oocytes in nests in ovarian tissues cultured in the presence of DEHP (Control= 
a

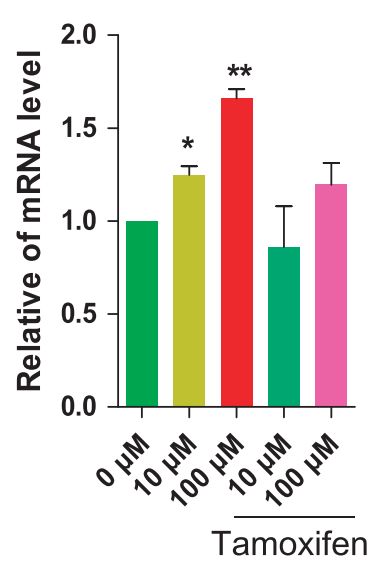

$E R \beta$

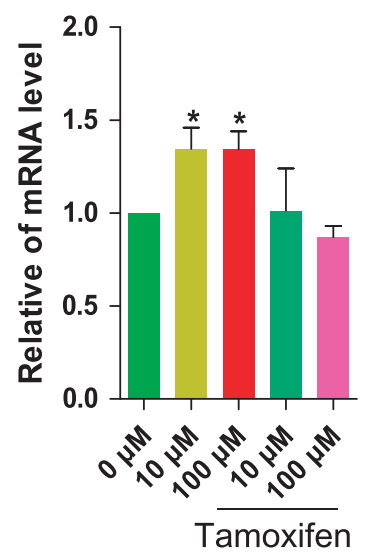

PPAR $\alpha$

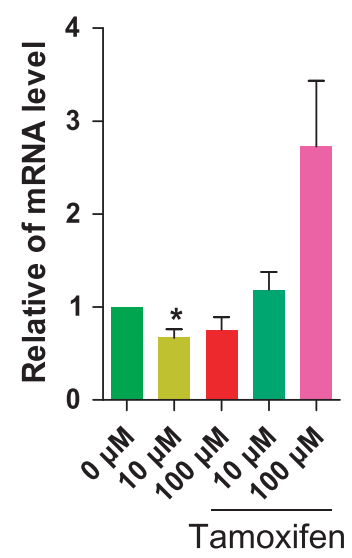

b

C
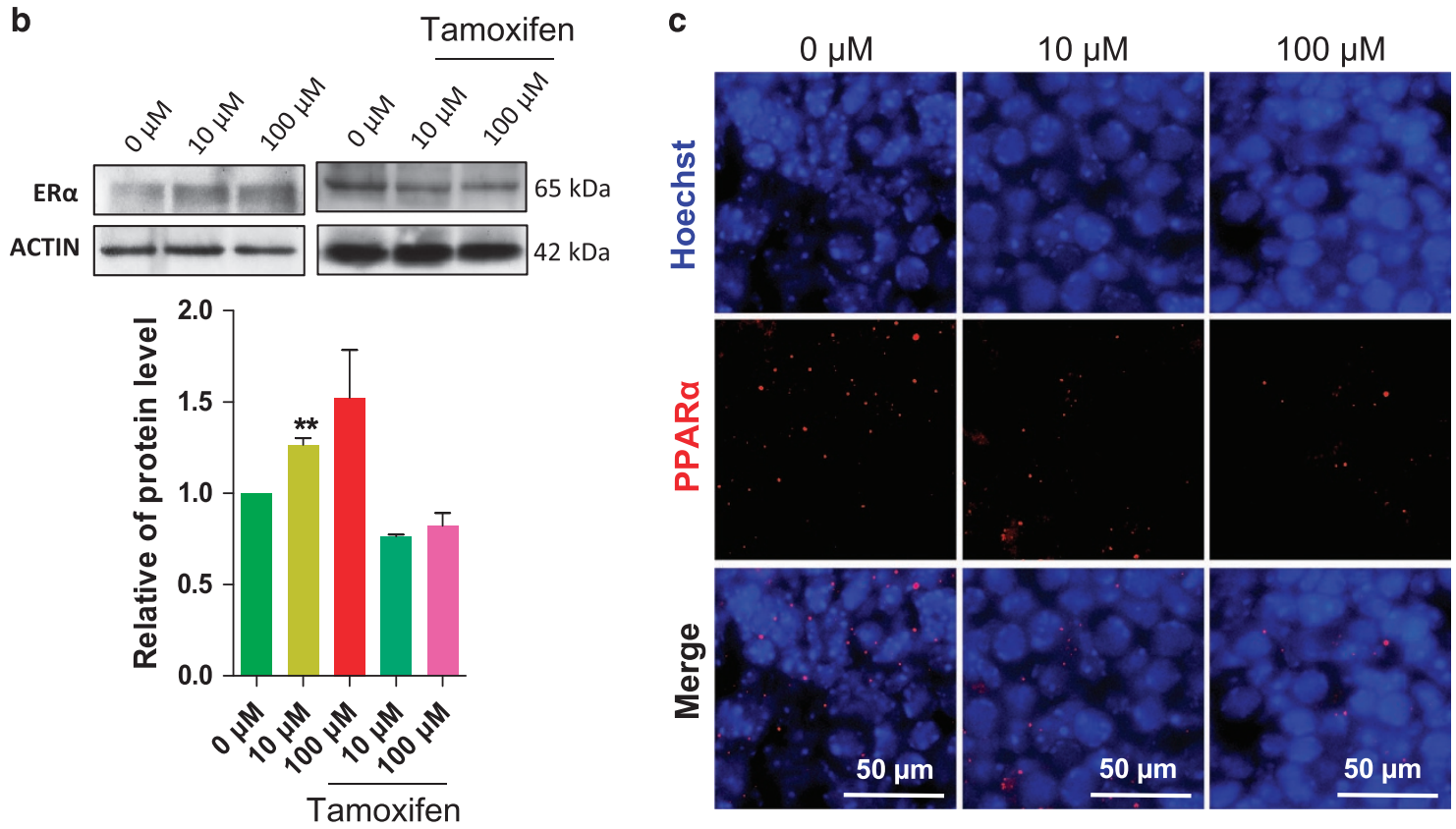

Figure 5 DEHP exposure affects the expression of ER and PPAR $\alpha$ in the ovary. (a) Representative qRT-PCR for ER $\alpha, E R \beta$ and PPAR $\alpha$ transcripts in ovarian tissues cultured for 6 days in control, DEHP and DEHP plus tamoxifen. (b) Representative WB of ER $\alpha$ expression. (c) PPAR $\alpha$-staining of the ovarian tissues after 6 days of culture with or without DEHP. The percentage of each group is presented as mean \pm SD. All experiments were repeated at least three times. $\left({ }^{\star} P<0.05 ;{ }^{* \star} P<0.01\right)$

$61.12 \pm 8.06 \%, 10 \mu \mathrm{M}$ DEHP $=44.20 \pm 1.55 \%$ and $100 \mu \mathrm{M}$ $\mathrm{DEHP}=36.51 \pm 1.54 \%)$.

RNA microarray analysis of $\mathbf{1 2 . 5}$ dpc ovarian tissues exposed to DEHP. To gain a better understanding of the biological effects of DEHP on embryonic ovaries we performed an RNA microarray analysis on $12.5 \mathrm{dpc}$ ovarian tissues cultured for two days in the presence of DEHP. DEGs between the control group and DEHP exposed tissues were screened. We found a total of 206 upregulated and 54 downregulated genes following $10 \mu \mathrm{M} \mathrm{DEHP}$ treatment, and 1529 upregulated and 1421 genes downregulated following $100 \mu \mathrm{M}$ DEHP treatment (Figures 8a and b; Supplementary Table S2). Compared to the $10 \mu \mathrm{M}$ DEHP, 1474 genes were significantly upregulated and 1505 genes were significantly downregulated in the $100 \mu \mathrm{M}$ DEHP group (Figures 8a and b; Supplementary Table S2). As a whole, 225 genes were significantly upregulated and 85 genes were significantly downregulated between control and DEHP exposed groups (Figures $8 \mathrm{a}$ and b; Supplementary Table S2).

Gene ontology (GO) analysis of the DEGs showed that DEHP exposure mostly perturbed genes involved in apoptosis and gonadal development (Figure 8c). As shown in Supplementary Table S2 and 3, apoptotic and gonad development genes altered after DEHP exposure included Foxl2, Gm13237, Nkx2-1, Lhx9, and Peg10, Opa1, Krt18, Rock1, 2610018G03RIK, Id1, Rhob, Psme3, Pmaip1, Il24, Phlda1, and Elmo1.

Moreover, we found DEGs of DNA binding factors in both the $10 \mu \mathrm{M}$ DEHP treatment and control groups (Supplementary Figure S5A). The effects of DEHP exposure on the gene 
expression showed considerable dose-dependent effects. In particular, by comparing DEGs between the $10 \mu \mathrm{M}$ and $100 \mu \mathrm{M}$ DEHP groups, we found that the higher treatment mostly clustered in cell-cell communication and signal transduction, plasma membrane and extracellular matrix and ion channel functions (Supplementary Figure S5B).
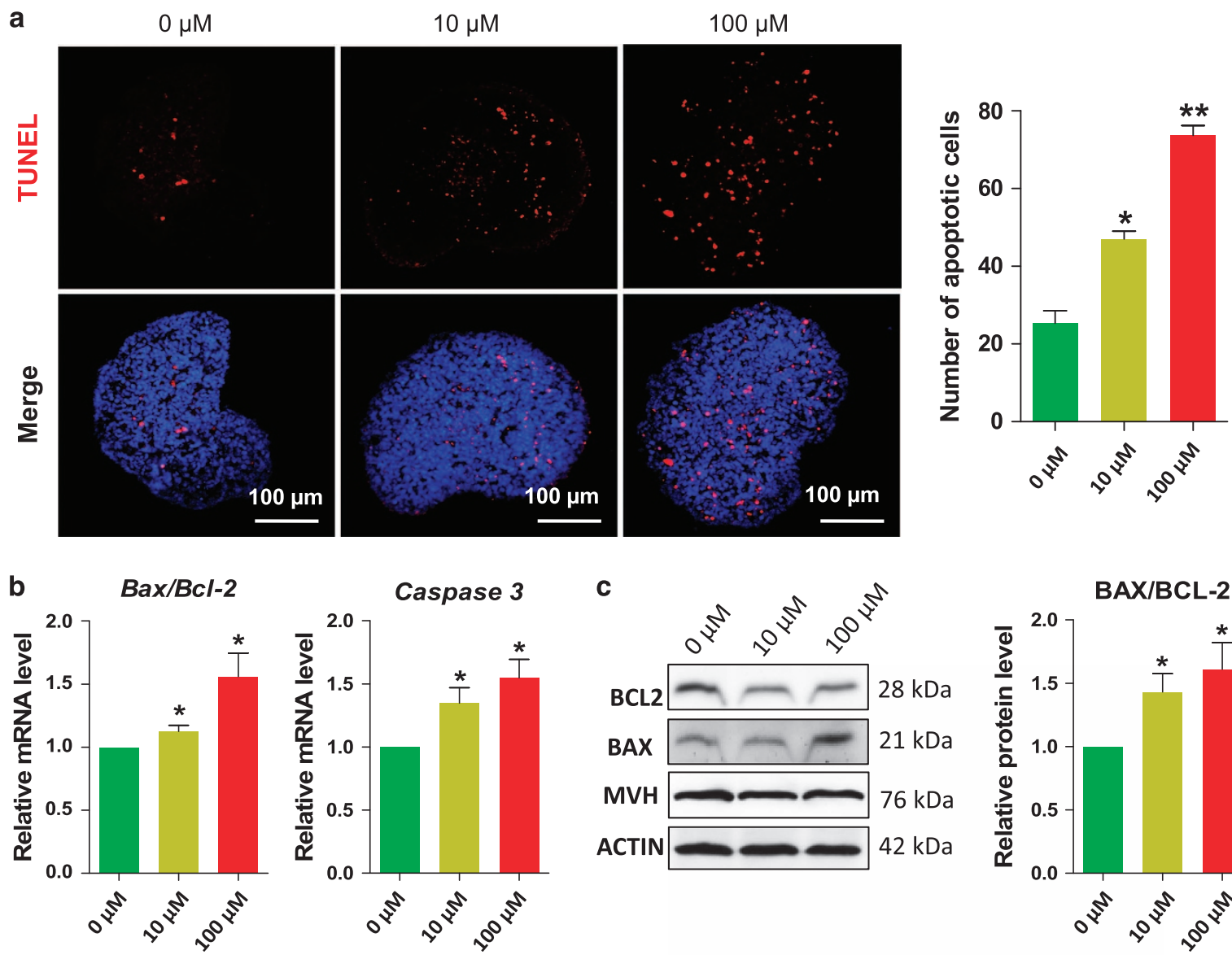

BAX/BCL-2
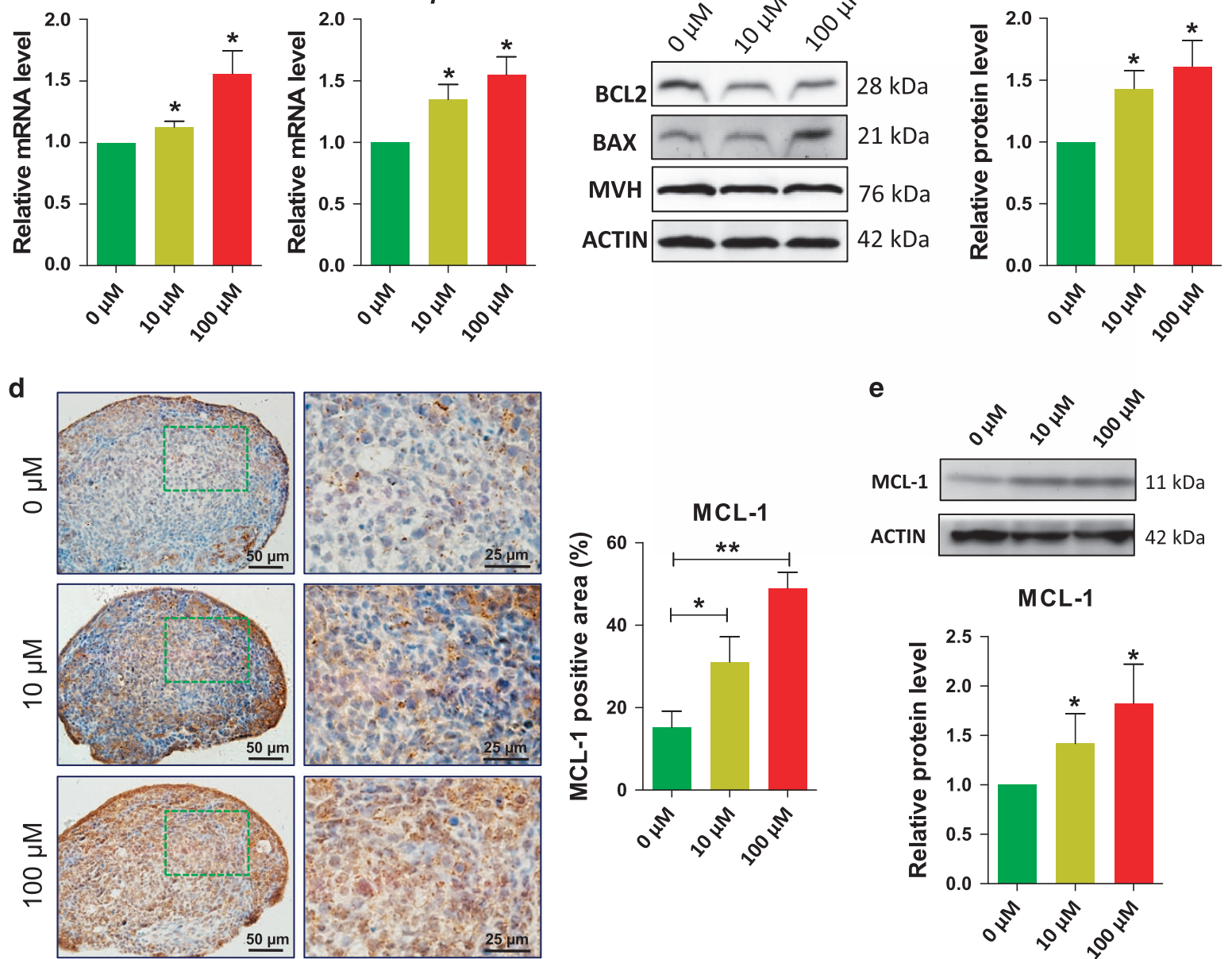

MCL-1

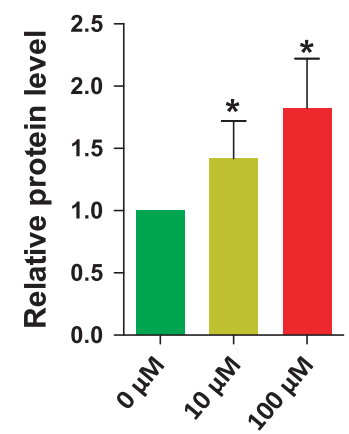

Figure 6 DEHP exposure promotes apoptosis in the ovary. (a) (Left) TUNEL-staining of the ovarian tissues after 6 days of culture with or without DEHP; (Right) Quantitative analyses of the number of TUNEL-positive cells. (b) Representative qRT-PCR for the apoptosis related genes Bax, Bcl-2 and Casp3 in ovarian tissues cultured for 6 days with or without DEHP. (c) (Left) representative WB of BAX and BCL-2; (Right) Quantitative analysis of BAX/BCL2 expression. (d) (Left) MCL-1-staining of the ovarian tissues after 6 days of culture with or without DEHP; (Right) Percentages of MCL-1 positive area. (e) (Up) Representative WB of MCL-1; (Down) Quantitative analysis of MCL-1 expression. The percentage of each group is presented as mean \pm SD. All experiments were repeated at least three times. $\left({ }^{*} P<0.05\right.$; $\left.{ }^{* *} P<0.01\right)$ 
a

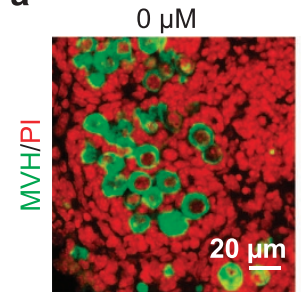

$10 \mu \mathrm{M}$

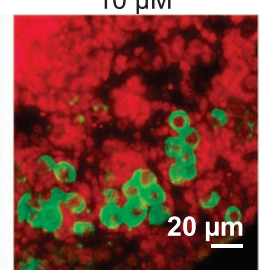

$100 \mu \mathrm{M}$

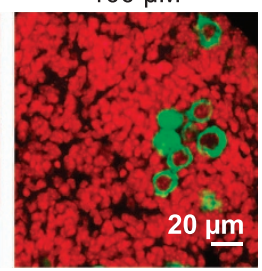

d $0 \mu \mathrm{M}$
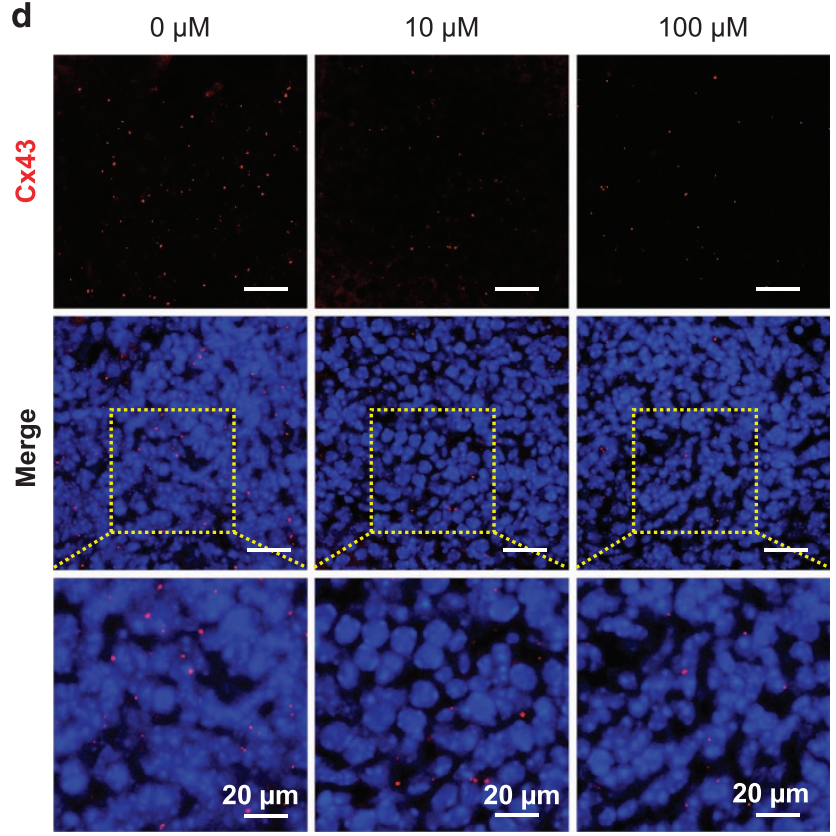

b
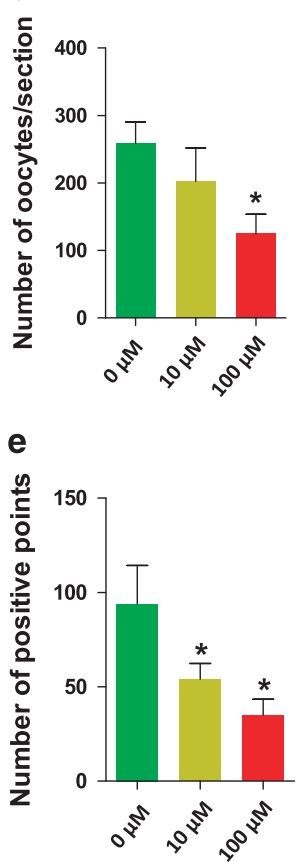

f

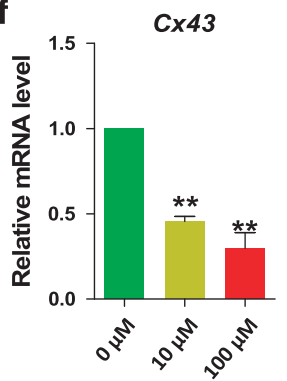

C

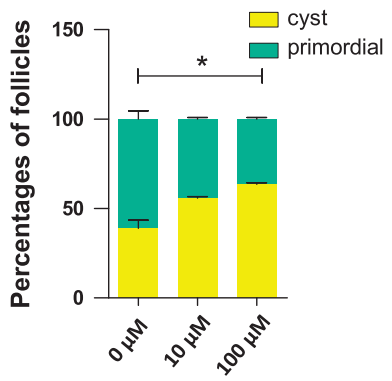

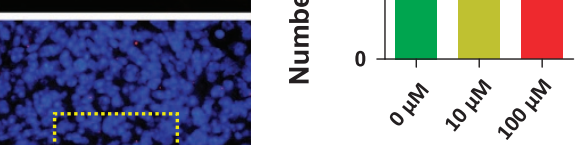

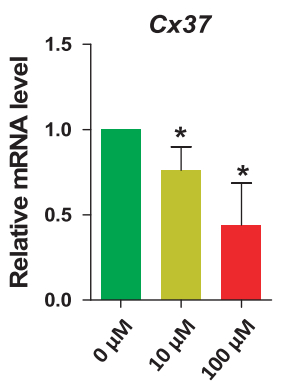

Figure 7 DEHP exposure causes a reduction of oocyte number and of cyst breakdown (a) Control and DEHP exposed ovarian tissue stained for MVH (green specific for oocytes) after 10 days DEHP exposure, nuclei red; bar is $20 \mu \mathrm{M}$. (b) Number of oocytes in control and DEHP exposed groups. (c) Percentage of oocytes in cysts and primordial follicles in control and DEHP exposed groups. DEHP impairs the expression of connexins, (d) Control and DEHP exposed ovarian tissues stained for Cx43 (red), nuclei blue; bar is $20 \mu \mathrm{M}$. (e) Number of $\mathrm{Cx} 43$ positive gap junction plaques in control and DEHP exposed ovarian tissues. (f) qRT-PCR for $C \times 43$ and $C \times 37$. Relative fold changes are presented as mean \pm SD. All experiments were repeated at least three times. $\left({ }^{*} P<0.05 ;{ }^{* *} P<0.01\right)$

\section{Discussion}

Several studies have reported that DEHP exposure can affect the correct development and functions of the ovary. Depending on the stage of development and exposure concentration and time, both in vivo and in vitro, DEHP has been shown to alter germ cell formation and development, meiotic initiation and progression, and primordial follicle assembly. ${ }^{10}$ The concentrations of 10-100 $\mu \mathrm{M}$ DEHP used in our study are in the range used by us and others in order to investigate the effect of this compound in vitro on some processes of oogenesis. ${ }^{17-19}$ They correspond to about 4 to $40 \mu \mathrm{g} / \mathrm{ml}$, a range reported in the blood of patients having a long-term exposure to DEHP-containing devices about 70 to $80 \mu \mathrm{g} / \mathrm{ml}$, or after neonatal exposure to DEHP following exchange transfusion with PVC catheters, between 13.2 to $84.9 \mu \mathrm{g} / \mathrm{ml}{ }^{20}$

We report here that in vitro exposure of the mouse fetal ovaries to DEHP inhibited the progression of oocytes throughout meiosis prophase I, specifically, the transition from the pachytene to the diplotene stage. Moreover, we found that oocytes exposed to DEHP showed increased DNA damage as evaluated by $\gamma \mathrm{H} 2 \mathrm{AX}$ and $\mathrm{BRCA} 1$ staining and the perdurability of RAD51 foci. As a consequence, defective oocytes could be prone to undergo apoptosis which is consistent with what we found in ovaries cultured in the presence of DEHP for six days.

Besides some morphological evidence in oocytes, we obtained indication of apoptosis in the ovarian cells following DEHP exposure from the increased $\mathrm{Bax} / \mathrm{BCl}-2$ ratio, Caspase 3 and $M C L-1$ expression. As a matter of fact, although MCL-1 is considered an anti-apoptotic protein, its expression has been reported to increase upon exposure to various types of apoptotic stimuli including DNA damage, likely as an attempt by cells to preserve their viability. ${ }^{21,22}$

Activation of a quality check point, at the pachytene stage, which is able to eliminate oocytes carrying DNA defects has been proposed as one of the causes of oocyte depletion occurring during prenatal oogenesis in mammals. ${ }^{23,24}$ Increased apoptosis explains the reduced number of oocytes scored by us after a prolonged culture period. Likewise, in vitro 

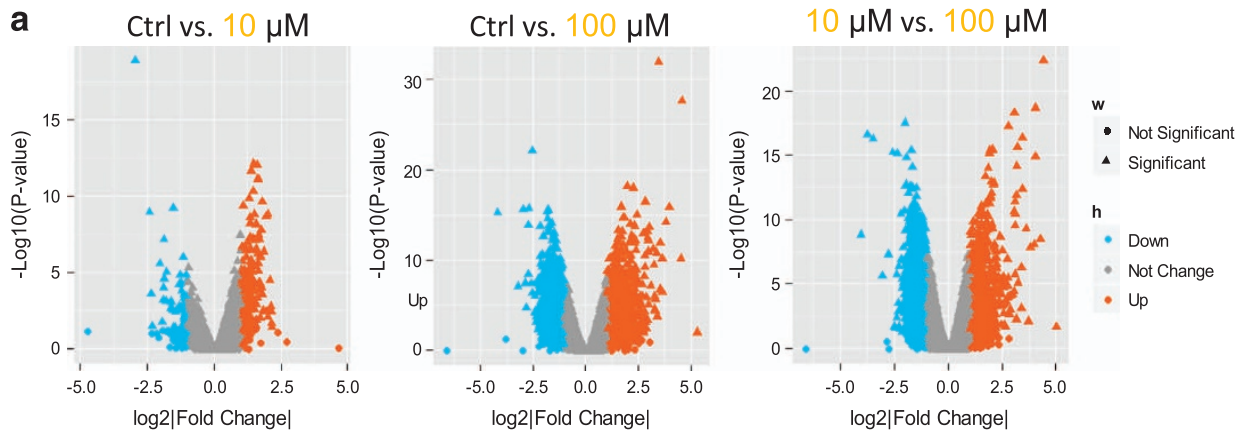

b

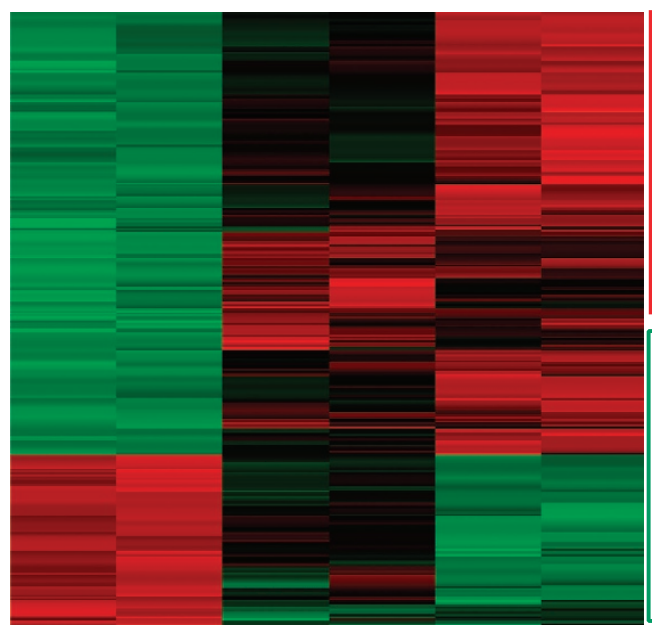

Up-regulated:

Mybl1, Tcf12, Krt18, Tnpo1,

Etnk1, Phf16, Mob1, Fam84b,

Plxnc1, Man1a, Impact,

Zfp395, Plxna4, Pik3c2a,

Dhcr7, Lhx9, AW549877,

Peg10, Phf16, Zfp518a ......

Down-regulated:

Hdhd1a, Prg2, Csmd1, Bst2,

Car5b, Lamc2, Npc1l1,

Slc17a6, Kcnj4, Fbp1,

5730455P16Rik, Camp,

Foxl1, Il24,Mir193b,

Cnga3,Clstn3 ......

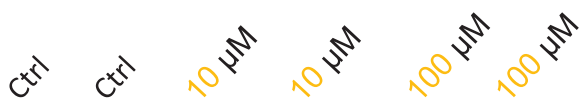

c Ctrl vs. DEHP treatment
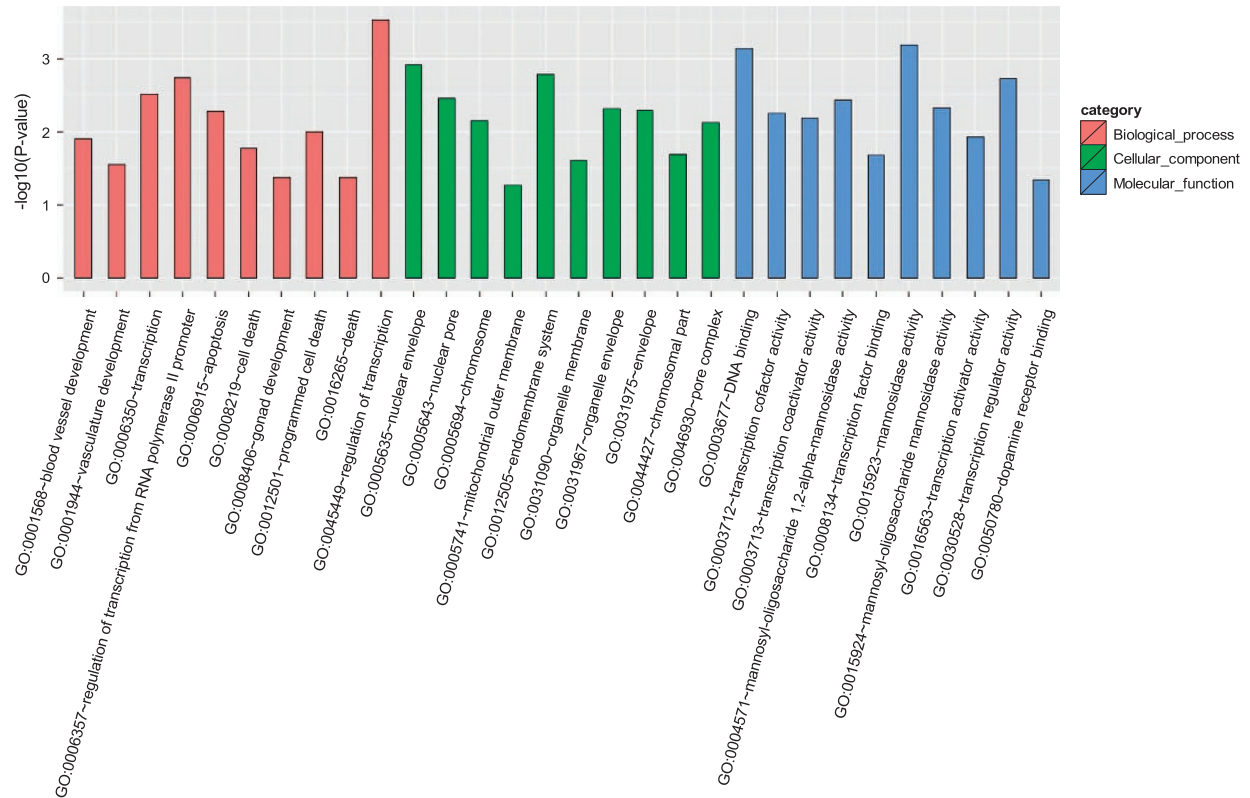

Figure 8 Microarray analyses of gene expression in $12.5 \mathrm{dpc}$ ovary cultured for 2 days in Control (Ctr) in the presence of DEHP. (a) Scatter plots of differentially expressed genes between Ctr and $10 \mu \mathrm{M}$ DEHP, control and $100 \mu \mathrm{M}$ DEHP, $10 \mu \mathrm{M}$ and $100 \mu \mathrm{M}$ DEHP groups. (b) Heatmap of differentially expressed genes between control and DEHP treated groups. (c) Gene ontology (GO) enrichment analysis of differentially expressed genes between Control (Ctr) and DEHP groups 
exposure for $24 \mathrm{~h}$ to MEHP, the active metabolite of DEHP, was shown to decrease the viability of mouse fetal oocytes, an effect attributed to an increase in oocyte oxidative stress. ${ }^{25}$

Since DEHP can interact with a subset of PPARs, a group of nuclear receptors that function as transcription factors for genes encoding enzymes involved in oxidative stress, ${ }^{1,26,27}$ it can be postulated that the increased DNA damage detected in cultured oocytes exposed to DEHP results from reduced activity of antioxidant enzymes. Our finding that ovarian tissues express PPARs and that such expression decreased in culture in the presence of DEHP support such a possibility. Alternatively, some of the DEHP effects reported here could be attributed to an estrogenic endocrine disruptor action. Actually, ERs are another possible DEHP targets. ${ }^{10}$ Two main classes of ER activated with different efficiency by E2 have been identified: ER $a$ and ER $\beta$, which are members of the nuclear hormone family of intracellular receptors. Once activated by E2, ERs are able to translocate into the nucleus and bind to DNA to regulate the activity of different genes. It is known that ER $a$ is expressed both by primordial germ cells and somatic cells of mouse sex indifferent gonads and that this expression continues to later stages while at $16.5 \mathrm{dpc} E R \beta$ expression was observed in both ovaries and testes. $8,9,28,29$ Here, we confirmed ER expression in the ovarian tissues regardless whether in somatic cells, oocytes or both and report an increased expression of both receptors in culture in the presence of DEHP. It is of note that in a previous study, we reported that the meiotic progression of fetal oocytes was delayed following the exposure of the pregnant mice to DEHP. This effect, resembling the present results, was associated to a reduction of the mRNA and protein expression of the meiosisspecific gene Stra8 while the DNA methylation level of the gene was increased. ${ }^{13}$ The capability of DEHP to perturb methylation of meiotic genes such as Stra8 and other genes in oocytes of neonatal mouse ovaries such as $L h x 8$ and of the maternal imprinted genes Igf2r and Peg3 in a transgenerational way, ${ }^{17}$ can be seen in the context of the contribution by ERs signaling to epigenetic changes. Several acetylases/deacetylases and methylases/demethylases can interact directly or indirectly with ER $a$ and cause histone modifications. ${ }^{30}$ In fact, in previous studies, using the same culture system, we found that Notch signaling dependent Stra8 methylation was necessary for correct meiotic progression. ${ }^{31}$ Moreover, ablation of G9a, a major mammalian H3K9 methyltransferase, showed that epigenetic gene silencing was crucial for proper meiotic prophase progression beyond the pachytene stage. ${ }^{32}$ Interestingly, ER $a$ and ER $\beta$ are present also in the human fetal ovary were they are present until the 20th week of gestation, when the expression of ERa substantially increases. ${ }^{33}$

It is to be mentioned here that exposure of mice from midgestation until birth to daily doses of bisphenol (BPA), a compound that like DEHP is considered an estrogenic endocrine disruptor, resulted in chromosome synaptic abnormalities and increased rates of recombination between homologous chromosomes in the mouse oocytes. Interestingly, these effects resembled those observed in mice homozygous for a targeted disruption of the gene encoding for $E R \beta^{34}$ Increased recombination was also observed in oocytes of rhesus monkeys prenatally exposed to BPA and in human fetal oocytes treated in vitro with BPA. ${ }^{35,36}$ Moreover, the expression of genes involved in recombination and DNA repair and the epigenetic integrity was altered in BPA-exposed mouse and human oocytes. ${ }^{7,37,38}$

In addition to effects on meiosis, the adverse effect reported by us in the present paper on gap junction assembly and germ cell cyst breakdown can be also be attributed to an estrogenic endocrine disruptor action. In fact, estrogens are able to modulate the quantity, size and distribution of gap junctions in the ovaries and to negatively affect germ cell cyst breakdown. ${ }^{17,39,40}$

Finally, the analysis of the RNA microarray data reinforced the notion of pleiotropic dose-dependent effect of DEHP on the mammalian ovary, highlighting the possibility that this compound can affect the expression of several genes involved beside in apoptosis, in a number of process guiding prenatal gonadal development.

\section{Materials and methods}

Reagents. Unless otherwise indicated, all chemicals were purchased from Sigma-Aldrich (Saint Louis, MO, USA). Stock solutions of DEHP were prepared using dimethylsulfoxide (DMSO) as the solvent at concentrations of 0.127 or $1.27 \mathrm{M}$ allowing an equal volume to be added to culture medium for each experimental groups. Tamoxifen, an ER antagonist, was dissolved in 50\% ethanol and was added to the culture medium at the final concentrations of $1 \mu \mathrm{M}$, and only $0.05 \%$ ethanol was present in the medium.

Animals. The procedures involving animals followed the regulations of the Ethics Committee of Qingdao Agriculture University, Shandong, China. Mice used in this experiment were of $\mathrm{CD} 1$ strain purchased from Vital River Laboratory Animal Technology Co. LTD (Beijing, China). Mice were housed in $21-22^{\circ} \mathrm{C}, 12 \mathrm{~h}$ dark $/ 12 \mathrm{~h}$ light cycles (lights on at 7:30) with free access to food and water. Female and male mice were paired together and checked for the presence of a vaginal plug the next morning. The day when the vaginal plug was detected was considered 0.5 day post coitum (d.p.c.).

Isolation and culture of the ovarian tissues. Isolation and culture of the ovarian tissues from $12.5 \mathrm{dpc}$ embryos were performed as previously described. ${ }^{12}$ In particular, after isolation, the ovaries were dissected in half and each piece was cultured in $600 \mu \mathrm{l}$ of $\alpha$-minimal essential medium ( $\alpha$-MEM; Hyclone, SH30265.01B, Beijing, China), supplemented with $10 \%$ fetal bovine serum (FBS; Gibco, 10099141, USA), $0.23 \mathrm{mM}$ sodium pyruvate (Hyclone, SH40003-12), $100 \mathrm{IU} / \mathrm{ml}$ of penicillin $\mathrm{G}$, and $100 \mathrm{mg} / \mathrm{ml}$ of streptomycin sulfate, $10 \mathrm{mIU} / \mathrm{ml}$ follicle stimulating hormone (FSH; RD, 5925-FS, MN, USA). In each well, $300 \mu$ l of medium was changed every two days and the culture carried out in a humidified incubator in $5 \%$ $\mathrm{CO}_{2}$ in air at $37^{\circ} \mathrm{C}$ for the indicated time (Supplementary Figure S1).

According to United States Food and Drug Administration (FDA), tolerable intake values for DEHP are $0.6 \mathrm{mg} / \mathrm{kg}$ bodyweight/day for parenteral exposure and $0.04 \mathrm{mg} /$ $\mathrm{kg}$ bodyweight/day for oral exposure, mouse ovaries were cultured in vitro and exposed to DEHP (Sigma, 36735-1G) at dose of $10 \mu \mathrm{M}$ or $100 \mu \mathrm{M}$. DEHP was dissolved in $0.1 \% \mathrm{DMSO}$, and $0.1 \% \mathrm{DMSO}$ alone as a vehicle control, respectively. After DEHP treatment, the ovaries were collected and kept for further analysis. ${ }^{10}$

Staining of meiotic chromosomes. Ovarian tissues were incubated in Hypo extraction buffer (HEB; 0.6 M Tris, $0.5 \mathrm{M}$ Sucrose, $0.17 \mathrm{M}$ trisodium citrate dehydrate, $0.5 \mathrm{M}$ EDTA, 0.5 M DTT, 0.1 M PMSF) for $1.5 \mathrm{~h}$ at room temperature. Tissues were then mechanically dispersed and cells transferred onto a slide fixed with $1 \%$ PFA overnight. Blocking was performed by dipping the slides in antibody dilution buffer (ADB; $0.3 \%$ BSA, 10\% normal goat serum and $0.005 \%$ Triton-X-100 in TBS) for 30 min before incubation in primary antibody, anti-SCP3 at a dilution of 1:150 (Abcam, ab97672, San Francisco, CA, USA; or Novus, NB300-232, Littleton, CO, USA), anti- $\gamma$ H2AX at a dilution of 1:150 (Abcam, ab26350), anti-RAD51 at a dilution of 1:150 (Abcam, ab133534), BRCA1 at a dilution of 1:100 (Boster, PB9015, Wuhan, China), or anti-MLH1 at a dilution of 1:150 (BD Pharmingen, 551091, Franklin Lakes, NJ, USA ) for $3 \mathrm{~h}$ at $37^{\circ} \mathrm{C}$. The slides were further fixed with ADB overnight at $4{ }^{\circ} \mathrm{C}$, washed three times with TBS, before incubation in secondary antibody (Beyotime, A0521 or A0516, Nantong, China; or Sigma, F0382 or F9006) 
at a dilution of 1:150 dilution in $\mathrm{ADB}$ for $3 \mathrm{~h}$ at $37^{\circ} \mathrm{C}$. Hoechst 33342 was used to stain nuclei and the slides were mounted with Vectashield (Vector, $\mathrm{H}-1000$, Shanghai, China).$^{41}$ Images were taken under a fluorescence microscope (BX51; Olympus, Tokyo, Japan).

Immunofluorescence. Ovaries tissues were fixed in 4\% paraformaldehyde (Solarbio, Beijing, China) for $12 \mathrm{~h}$ and processed for standard $5 \mu \mathrm{m}$ thick histological sections. Before staining, slides were incubated in $0.01 \mathrm{M}$ sodium citrate at $96{ }^{\circ} \mathrm{C}$ for 10 min and blocked with BDT ( $3 \%$ BSA, $10 \%$ normal goat serum in TBS) for $45 \mathrm{~min}$. and incubated with primary antibodies, rabbit anti-MVH antibody (1:150; Abcam, ab13840), rabbit anti-CX43 antibody (1:150) (Abcam, ab11370), rabbit anti-PPAR $\alpha$ (Sangon, D161086, Shanghai, China), overnight at $4{ }^{\circ} \mathrm{C}$. After careful washing with PBS, sections were incubated with $\mathrm{CY} 3$-conjugated goat antirabbit secondary antibody at 1:150 dilution (Beyotime, A0516, Nantong, China) at $37^{\circ} \mathrm{C}$ for $30 \mathrm{~min}$. Vectashield (Vector) was used to mount the slides. Images were taken under a fluorescence microscope (BX51; Olympus, Tokyo, Japan).

Immunohistochemistry. MCL-1 proteins were localization using immunohistochemistry $(\mathrm{IHC})$. Put the slides in $0.01 \mathrm{M}$ sodium citrate at $96^{\circ} \mathrm{C}$ for $10 \mathrm{~min}$, then in $3 \% \mathrm{H}_{2} \mathrm{O}_{2}$ for 10 min and blocked with BDT (3\% BSA, 10\% normal goat serum in TBS) for 45 min. Rehydrated sections were incubated with MCL-1 (Sangon, D261457) primary antibodies overnight at $4^{\circ} \mathrm{C}$, then reacted with biotinlabeled secondary antibodies for $40 \mathrm{~min}$ at room temperature and finally stained using DAB peroxidase substrate and counterstained with hematoxylin. Image Pro Plus software 6.0 (Media Cybernetics, Rockville, MD, USA) was used to count the positive cells. In each ovary, five non-redundant sections were selected for counting and at least three ovaries per experimental group was analyzed. The data were collected from at least nine mice per treatment, and calculated the DAB peroxidase stained (positive) region, hematoxylin stained (negative) region and defined the positive region as positive stained region/(positive+negative stained regions).

TUNEL staining. TUNEL staining was performed using the Roche "In Situ Cell Death Detection Kit" (Roche, 12156792910, Mannheim, Germany). Paraffin sections were heated at $60{ }^{\circ} \mathrm{C}$ for $2 \mathrm{~h}$, washed in xylene and rehydrated through a series of ethanol and double distilled water. Before staining sections were treated with proteinase $\mathrm{K}$ for $15 \mathrm{~min}$ at room temperature, and carefully washed three times with PBS. $50 \mu$ of TUNEL reaction mixture (Enzyme Solution and Label Solution; 1:9 dilution) were added to the samples. For negative control, $50 \mu$ l label solution (without terminal transferase) instead of TUNEL reaction mixture, was added to the slides. All slides were incubated in a humidified atmosphere for 60 min at $37^{\circ} \mathrm{C}$ in the dark. Nuclei were counterstained with Hoechst 33342 (Beyotime). Images were taken under a fluorescence microscope (BX51; Olympus).

TEM observations. For transmission electron microscopic (TEM) analyses, control and DEHP treated ovaries tissues were fixed in $2.5 \%$ glutaraldehyde in 0.1 M PBS at pH 7.2 at $4{ }^{\circ} \mathrm{C}$ for $24 \mathrm{~h}$ and processed following standard methods. Sectioning was performed using Leica ultra-microtome (Leica EM UC7, Wetzlar, Germany) equipped with a diamond knife. Ultrathin sections of $70 \mathrm{~nm}$ were collected and plated on form var-coated, carbon-stabilized copper grids, the samples were further stained with uranyl acetate and lead citrate. Samples were observed using a HITACHI HT7700 transmission electron microscope (HITACHI, Tokyo, Japan) with an accelerating voltage of $80 \mathrm{kV}$.

Western blotting. Total proteins were extracted with RIPA lysis solution (Beyotime) for $30 \mathrm{~min}$ on ice with frequent vortexing. $20 \mu \mathrm{l}$ protein solution was mixed with $5 \mu$ l of sodium dodecyl sulfate-polyacrylamide gel electrophoresis (SDSPAGE) sample loading buffer and boiled for $5 \mathrm{~min}$. Lysates were collected by centrifugation (12 $000 \mathrm{rpm}$ for $5 \mathrm{~min}$ ). Proteins were separated by SDS-PAGE with a $4 \%$ stacking gel and a $10 \%$ separating gel for $2 \mathrm{~h}$ at $100 \mathrm{~V}$ and then transferred onto polyvinylidene fluoride membrane ( $18 \mathrm{~min}, 15 \mathrm{~V}$ ) by electrophoresis. The membrane was blocked in TBST (Tris-buffered saline with Tween-20) with $10 \%$ BSA at $4{ }^{\circ} \mathrm{C}$ for $4 \mathrm{~h}$ and incubated with the rabbit anti-DAZL antibody (1:1000) (Abcam, ab34139), rabbit anti-STRA8 antibody (1:1000) (Abcam, ab49602), rabbit anti-SCP3 antibody (1:1000) (Novus, NB300-232), rabbit anti-MVH antibody (1:1000) (Abcam, ab13840), rabbit anti-BCL2 antibody (1:1000) (ImmunoWay, YTO470, Newark, NJ, USA), rabbit anti-BAX antibody (1:1000) (Cell Signaling Technology, 2772 Shanghai, China), rabbit anti-MCL-1 antibody (1:300), mouse anti- $\gamma \mathrm{H} 2 \mathrm{AX}$ antibody (1:1000) (Abcam, ab26350), mouse anti- estrogen receptors (ER) $\alpha$ antibody (1:500) (Abcam, ab2746), mouse anti- $\beta$-actin antibody (1:1000) (Boster, BM0627) in TBST buffer containing $5 \%$ bovine serum albumin (BSA) (Solarbio, A8020) overnight at $4^{\circ}$ C. Then membranes were washed three times in TBST and incubated with horseradish peroxidase (HRP)-conjugated goat anti-mouse lgG (Beyotime, A0216) at 1:2000 dilution in TBST. The BeyoECL plus kit (Beyotime, P0018) was used for signal development. Beta-actin was used as the loading control. The intensity of the signal was quantified using the AlphaView SA software.

RNA extraction and quantitative RT-PCR. Total RNA was extracted using the RNAprep pure Micro Kit (Aidlab, RN28, Beijing, China) according to the manufacturer's instructions. Reverse transcription was performed using the TransScript One-Step gDNA Removal Kit and cDNA Synthesis Kit (TransGen Biotech, AT311, Beijing, China). All primers used in this research are listed in Supplementary Table S1. Relative quantification analysis was carried out with the LightCycler 480 II (Roche) using the LightCycler 480 SYBR Green I Master Kit (Roche, 04887352001) according to the manufacturer's instructions. Each sample contained three technical replicates and reactions were performed with three biological replicates. The PCR conditions were as follows: $10 \mathrm{~min}$ at $95^{\circ} \mathrm{C}$, followed by 45 cycles of $95^{\circ} \mathrm{C}$ for $10 \mathrm{~s}, 60^{\circ} \mathrm{C}$ for $30 \mathrm{~s}$ and $72{ }^{\circ} \mathrm{C}$ for $20 \mathrm{~s}$. Gene expression levels were calculated using $\beta$-actin for normalization. Relative transcript abundance was calculated using the $2^{-\Delta \Delta C T}$ method. ${ }^{41}$ Data were expressed as mean $\pm S D$ and calculated from at least three times independent replicates.

RNA microarray. The protocols of total RNA microarray hybridization were consistent with our previous study. ${ }^{42}$ Briefly, the integrity and concentration of total RNA were measured by the Agilent 2100 Bioanalyzer (Agilent Technologies, Santa Clara, CA, USA). We used $6 \mu \mathrm{g}$ of high-quality RNA labeled with Cy5, and hybridized to a mouse oligo microarray (Phalanx Mouse Whole Genome One Array; Phalanx Biotech Group, Palo Alto, CA, USA). Each array contained 26423 DNA oligonucleotide probes of the sense strand. After hybridization, the fluorescent signals on the array were scanned using an Axon 4000 (Molecular Devices, Sunnyvale, CA, USA). Data analysis was performed based on the manufacturer's instructions. The Differential Expression Genes (DEGs) were defined as log2 Ifold changel $>1$ (absolute Ifold changel $>2$ ) and $P<0.05$. For Gene Ontology enrichment analysis, we used the DAVID software (https://david.ncifcrf.gov/). Each array was performed using tissues from 16 ovaries for every experimental groups, and the analysis repeated at least two times.

Statistical analysis. For each set of results, independent trials were repeated at least three times; data were represented as mean \pm SD. Differences among groups were statistically tested by Student's $t$-test or one-way analysis of variance (ANOVA) multiple comparisons by using Graph-Pad Prism analysis software. Comparisons were considered significant at ${ }^{\star} P<0.05$ and highly significant at ${ }^{* *} P<0.01$.

\section{Conflict of Interest}

The authors declare no conflict of interest.

Acknowledgements. This work was supported by National Key Research and Development Program of China (2016YFD0501207), National Nature Science Foundation of China (31572225 and 31471346), Science and Technology Development Plan Project of Medicine and Health Department of Shandong Province (2016WS0627), Research Program of Wuhan Polytechnic University and Jining Medical University (2016y30 and JY2016KJ041Y) and the Fund for Doctoral Scientific Research Startup of Qingdao Agricultural University (6631116019).

\section{Publisher's Note}

Springer Nature remains neutral with regard to jurisdictional claims in published maps and institutional affiliations.

1. Lovekamp-Swan T, Davis BJ. Mechanisms of phthalate ester toxicity in the female reproductive system. Environ Health Perspect 2003; 111: 139-145.

2. Zhang XF, Zhang LJ, Li L, Feng YN, Chen B, Ma JM et al. Diethylhexyl phthalate exposure impairs follicular development and affects oocyte maturation in the mouse. Environ $\mathrm{Mol}$ Mutagen 2013; 54: 354-361.

3. Engel SM, Wolff MS. Causal inference considerations for endocrine disruptor research in children's health. Annu Rev Public Health 2013; 34: 139-158.

4. Lai FN, Liu JC, Li L, Ma JY, Liu XL, Liu YP et al. Di (2-ethylhexyl) phthalate impairs steroidogenesis in ovarian follicular cells of prepuberal mice. Arch Toxicol 2017; 91: 1279-1292. 
5. Li L, Liu JC, Zhao Y, Lai FN, Yang F, Ge W et al. Impact of diethylhexyl phthalate on gene expression and development of mammary glands of pregnant mouse. Histochem Cell Biol 2015; 144: 389-402.

6. Hannon PR, Flaws JA. The effects of phthalates on the ovary. Front Endocrinol (Lausanne) 2015; 6 : 8.

7. Krotz SP, Carson SA, Tomey C, Buster JE. Phthalates and bisphenol do not accumulate in human follicular fluid. J Assist Reprod Genet 2012; 29: 773-777.

8. La Sala G, Farini D, De Felici M. Rapid estrogen signalling in mouse primordial germ cells. Exp Cell Res 2010; 316: 1716-1727.

9. Moe-Behrens GH, Klinger FG, Eskild W, Grotmol T, Haugen TB, De Felici M. Akt/PTEN signaling mediates estrogen-dependent proliferation of primordial germ cells in vitro. $\mathrm{Mol}$ Endocrinol 2003; 17: 2630-2638.

10. Zhang T, Shen W, De Felici M, Zhang XF. Di(2-ethylhexyl)phthalate: Adverse effects on folliculogenesis that cannot be neglected. Environ Mol Mutagen 2016; 57: 579-588.

11. Li L, Zhang T, Qin XS, Ge W, Ma HG, Sun LL et al. Exposure to diethylhexyl phthalate (DEHP) results in a heritable modification of imprint genes DNA methylation in mouse oocytes. Mol Biol Rep 2014; 41: 1227-1235.

12. Zhang ZP, Liang GJ, Zhang XF, Zhang GL, Chao HH, Li L et al. Growth of mouse oocytes to maturity from premeiotic germ cells in vitro. PLOS ONE 2012; 7: e41771.

13. Zhang XF, Zhang T, Han Z, Liu JC, Liu YP, Ma JY et al. Transgenerational inheritance of ovarian development deficiency induced by maternal diethylhexyl phthalate exposure. Reprod Fertil Dev 2015; 27: 1213-1221.

14. Rogakou EP, Nieves-Neira W, Boon C, Pommier Y, Bonner WM. Initiation of DNA fragmentation during apoptosis induces phosphorylation of H2AX histone at serine 139. $J$ Biol Chem 2000; 275: 9390-9395.

15. Keeney S. Spo11 and the formation of DNA double-strand breaks in meiosis. Genome Dyn Stab 2008; 2: 81-123.

16. Chen J. The cell-cycle arrest and apoptotic functions of p53 in tumor initiation and progression. Cold Spring Harb Perspect Med 2016; 6: a026104.

17. Zhang T, Li L, Qin XS, Zhou Y, Zhang XF, Wang LQ et al. Di-(2-ethylhexyl) phthalate and bisphenol $\mathrm{A}$ exposure impairs mouse primordial follicle assembly in vitro. Environ $\mathrm{Mol}$ Mutagen 2014; 55: 343-353.

18. Mu X, Liao X, Chen X, Li Y, Wang M, Shen $C$ et al. DEHP exposure impairs mouse oocyte cyst breakdown and primordial follicle assembly through estrogen receptor-dependent and independent mechanisms. J Hazard Mater 2015; 298: 232-240.

19. Ambruosi B, Uranio MF, Sardanelli AM, Pocar P, Martino NA, Paternoster MS et al. In vitro acute exposure to DEHP affects oocyte meiotic maturation, energy and oxidative stress parameters in a large animal model. PLOS ONE 2011; 6: e27452.

20. Kavlock R, Boekelheide K, Chapin R, Cunningham M, Faustman E, Foster P et al. NTP center for the evaluation of risks to human reproduction: phthalates expert panel report on the reproductive and developmental toxicity of di(2-ethylhexyl) phthalate. Reprod Toxicol 2002; 16: 529-653.

21. Hutt KJ. The role of $\mathrm{BH} 3$-only proteins in apoptosis within the ovary. Reproduction 2015; 149 : R81-R89.

22. Ke F, Voss A, Kerr JB, O'Reilly LA, Tai L, Echeverry $\mathrm{N}$ et al. BCL-2 family member BOK is widely expressed but its loss has only minimal impact in mice. Cell Death Differ 2012; 19: 915-925.

23. Felici MD, Klinger FG. DNA Damage and Apoptosis in Fetal and Ovarian Reserve Oocytes. Springer: Netherlands, 2011.

24. Li XC, Schimenti JC. Mouse pachytene checkpoint 2 (trip13) is required for completing meiotic recombination but not synapsis. PLoS Genet 2007; 3: e130.

25. Bonilla E, del Mazo J. Deregulation of the Sod1 and Nd1 genes in mouse fetal oocytes exposed to mono-(2-ethylhexyl) phthalate (MEHP). Reprod Toxicol 2010; 30: 387-392.

26. Lovekamp TN, Davis BJ. Mono-(2-ethylhexyl) phthalate suppresses aromatase transcript levels and estradiol production in cultured rat granulosa cells. Toxicol Appl Pharmacol 2001 172: 217-224.

27. Wang W, Craig ZR, Basavarajappa MS, Gupta RK, Flaws JA. Di (2-ethylhexyl) phthalate inhibits growth of mouse ovarian antral follicles through an oxidative stress pathway. Toxicol Appl Pharmacol 2012; 258: 288-295.
28. Jefferson WN, Couse JF, Banks EP, Korach KS, Newbold RR. Expression of estrogen receptor beta is developmentally regulated in reproductive tissues of male and female mice. Biol Reprod 2000; 62: 310-317.

29. Lemmen JG, Broekhof JL, Kuiper GG, Gustafsson JA, van der Saag PT, van der Burg B. Expression of estrogen receptor alpha and beta during mouse embryogenesis. Mech Dev 1999; 81: 163-167.

30. Moyal L, Lerenthal Y, Gana-Weisz M, Mass G, So S, Wang SY et al. Requirement of ATMdependent monoubiquitylation of histone $\mathrm{H} 2 \mathrm{~B}$ for timely repair of DNA double-strand breaks. Mol Cell 2011; 41: 529-542.

31. Feng YM, Liang GJ, Pan B, Qin XS, Zhang XF, Chen CL et al. Notch pathway regulates female germ cell meiosis progression and early oogenesis events in fetal mouse. Cell Cycle 2014; 13: 782-791.

32. Tachibana M, Nozaki M, Takeda N, Shinkai Y. Functional dynamics of H3K9 methylation during meiotic prophase progression. EMBO J 2007; 26: 3346-3359.

33. Vaskivuo TE, Maentausta M, Torn S, Oduwole O, Lonnberg A, Herva R et al. Estrogen receptors and estrogen-metabolizing enzymes in human ovaries during fetal development. $J$ Clin Endocrinol Metab 2005; 90: 3752-3756.

34. Susiarjo M, Hassold TJ, Freeman E, Hunt PA. Bisphenol A exposure in utero disrupts early oogenesis in the mouse. PLoS Genet 2007; 3: e5.

35. Hunt PA, Lawson C, Gieske M, Murdoch B, Smith H, Marre A et al. Bisphenol A alters early oogenesis and follicle formation in the fetal ovary of the rhesus monkey. Proc Natl Acad Sci USA 2012; 109: 17525-17530.

36. Brieno-Enriquez MA, Reig-Viader R, Cabero L, Toran N, Martinez F, Roig I et al. Gene expression is altered after bisphenol A exposure in human fetal oocytes in vitro. Mol Hum Reprod 2012; 18: 171-183.

37. Eichenlaub-Ritter U, Pacchierotti F, Bisphenol A. Effects on mammalian oogenesis and epigenetic integrity of oocytes: a case study exploring risks of endocrine disrupting chemicals. Biomed Res Int 2015; 2015: 698795.

38. Chao HH, Zhang XF, Chen B, Pan B, Zhang LJ, Li L et al. Bisphenol A exposure modifies methylation of imprinted genes in mouse oocytes via the estrogen receptor signaling pathway. Histochem Cell Biol 2012; 137: 249-259.

39. Risek B, Klier FG, Phillips A, Hahn DW, Gilula NB. Gap junction regulation in the uterus and ovaries of immature rats by estrogen and progesterone. J Cell Sci 1995; 108: 1017-1032.

40. Zhang $\mathrm{HQ}$, Zhang XF, Zhang LJ, Chao HH, Pan B, Feng YM et al. Fetal exposure to bisphenol $A$ affects the primordial follicle formation by inhibiting the meiotic progression of oocytes. Mol Biol Rep 2012; 39: 5651-5657.

41. Zhang XF, Zhang T, Wang L, Zhang HY, Chen YD, Qin XS et al. Effects of diethylhexyl phthalate (DEHP) given neonatally on spermatogenesis of mice. Mol Biol Rep 2013; 40: 6509-6517.

42. Li L, Liu JC, Lai FN, Liu HQ, Zhang XF, Dyce PW et al. Di (2-ethylhexyl) phthalate exposure impairs growth of antral follicle in mice. PLOS ONE 2016; 11: e0148350.

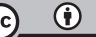

Cell Death and Disease is an open-access journal published by Nature Publishing Group. This work is licensed under a Creative Commons Attribution 4.0 International License. The images or other third party material in this article are included in the article's Creative Commons license, unless indicated otherwise in the credit line; if the material is not included under the Creative Commons license, users will need to obtain permission from the license holder to reproduce the material. To view a copy of this license, visit http://creativecommons.org/licenses/by/4.0/

(C) The Author(s) 2017

Supplementary Information accompanies this paper on Cell Death and Disease website (http://www.nature.com/cddis) 